\title{
FRÖMMIGKEIT UND MUSIK
}

Was ist Frömmigkeit? Laut dem Katechismus der katholischen Kirche besteht die Frömmigkeit (pietas) in der Gerechtigkeit gegenüber Gott ${ }^{1}$. Die drei Hauptbereiche der Frömmigkeit sind Almose, Gebet und Fasten. ${ }^{2}$ Von diesen drei kommt für die Musik das Gebet in Betracht. Das Gebet besteht in Anbetung, Danksagung, Lobpreis und Bitten. Musik in der Liturgie und bei unterschiedlichen Andachten soll die Frömmigkeit der Gläubigen befördern und unterstützen. Nur fromme Musik (musica pia) solle in den Kirchen erklingen, und die Kirche ist berechtigt festzulegen, welche Musik für den Gottesdienst geeignet sei.

Die Päpste und regionale Konzile äußerten mehrmals ihre Ansichten über die liturgisch korrekte Kirchenmusik und ihre Ausführung. Die umfangreichste und gründlichste Äußerung über Kirchenmusik vor dem Motu proprio von Papst Pius X. (1903) stellt die Enzyklika Annus qui vom Papst Benedikt XIV. aus dem Jahre 1749 dar. $^{3}$ Nach Karl Gustav Fellerer war gerade diese Enzyklika für die katholische Kirchenmusik der Klassik maßgebend. ${ }^{4}$ Deshalb halte ich Annus qui auch in Bezug auf das Thema des Colloquiums 2013 wichtig.

Seit dem Konzil von Trient sind viele Neuerungen auch in die Kirchenmusik übernommen worden. Die Mehrstimmigkeit (musica figuralis) ist fast zum Regel geworden. Nicht nur die Orgeln, sondern auch andere Musikinstrumente sind in den Kirchen sowohl in der Begleitung von Vokalmusik als auch im eigenständigen Spiel heimisch geworden. Darüber hinaus ist die dramatische Musik aus den Theatern in die Kirche eingedrungen. Benedikt XIV. war über diese Neuerungen nicht begeistert und versuchte in seiner Enzyklika, die Einflüsse der weltlichen Musik in der Kirchenmusik zu begrenzen. Im Vergleich mit der nach dem

1 Katechismus katolické cirkve, Praha, 1995, Paragraph 1807.

2 Mt 6, 2-18.

3 Für die Anschaffung einer Kopie dieses Dokumentes bin ich mit Dank Dr. Ing. Eduard Krumpolc CSc.,ThD. verbunden. Diese Enzyklika hat Benedikt XIV. anläßlich des Jubiläumsjahres 1750 herausgegeben. Von insgesamt 15 Paragraphen behandeln 13 die Kirchenmusik. 
zweiten Vatikanischen Konzil erschienenen oberflächlichen Instruktion Musica sacra $(1967)^{5}$ stützte sich die Encyklika Annus qui auf eine hohe Kenntnis der damaligen Fachliteratur. Benedikt XIV. beruft sich in seinem Text auf mehr als 70 Autoren seit Ambrosius († 397) und Augustinus (354-430) bis hin zu seinen Zeitgenossen Muratori (1672-1750) und Feijòo (1676-1764). Der Papst argumentiert sehr vorsichtig: Zuerst zitiert er die kirchlichen Autoren, die die Neuerungen abgelehnt haben. Erst dann beruft er sich auf Stimmen, die diese, wenn auch mit Vorbehalt, guthießen.

Die Vorstellung des Papstes über die rechte Kirchenmusik wurde grundlegend vom Mailänder Konzil (1565) beeinflußt: Die Gesänge und Klänge sollen ernst, fromm und dem Hause und Lobe Gottes angemessen sein, wobei die (gesungenen) Worte so verständlich sein sollten, dass durch sie die Zuhörer zur Frömmigkeit ermuntert würden. ${ }^{6}$ Streng hingegen verurteilte der Papst in Paragraph 3 jede Musik, die etwas Prophanes, Weltliches oder Theatralisches enthält. ${ }^{7}$ Was er unter dem Begriff mundanum versteht, definiert er allerdings nicht klar. Deutlicher ist der Begriff des Theatrale, unter dem er alle für das Theater geschaffene Musik versteht und für die Kirche unziemlich hält. Ob der Papst dabei amoralische Elemente in den Opernthemen oder die oft zweifelhafte Lebenshaltung der Opernsolisten im Sinne hatte, wird nicht explizit gesagt. Eine allgemeine Verurteilung des Theaters kam aber nicht in Betracht, da in der gleichen Zeit die kirchliche Hierarchie die Schuldramen der Jesuiten und der Piaristen unterstützte.

Obwohl das Wort Oper in der Enzyklika nicht einmal vorkommt, hatte der Papst bestimmt die Opernmusik seiner Zeit im Sinne gehabt. Benedikt XIV. (1675-1758) wurde in Bologna geboren, verbrachte aber die meisten Jahre in Rom. In den Jahren 1731-1740 war er Erzbischof von Bologna, wo damals drei Operntheater existierten, aber es ist zu bezweifeln, dass er als hoher kirchlicher Würdenträger die Oper in Bologna je besucht hat. ${ }^{8}$ Er konnte also die Oper aus eigener Wahrnehmung kaum kennenlernen, sondern seine Kenntnis über sie nur von den Berichtstattern haben.

Das Theatrale wurde jedoch schon lange vor der Entstehung der Oper von der Kirche für unziemlich gehalten. In diesem Punkt beruft sich der Papst auf den heiligen Hieronymus (4. Jahrhundert) ...theatrales moduli. Das Theatrale bezog sich ursprünglich nicht auf die Musik im Allgemeinem, sondern eher auf eine besondere Art des Vortrags. Nicht nur Hieronymus im 4. Jahrhundert, sondern auch spätere Kirchenautoritäten bezeichneten den theatralischen Gesang (trago-

5 Sie wurde mit Recht von Kirchenmusikkennern scharf kritisiert. Die Instruktion „Musicam sacram“ mit einem Kommentar von Higinio Anglès. Musicae sacrae ministerium 1967, Nr. 2-3, S. 37-47.

6 Paragraph 9: Cantus et soni graves sint, pii ac distincti, ad Domui Dei ac Divinis laudibus accommodati ut simul verba intelligantur et ad pietatem auditores excitantur.

8 MATTEUCCI, Benedetto. Il papato di fronte dell'assolutismo e al giurisdizionalismo (1605-1774). In I papi nella storia a cura di Pio Paschini-Vincenzo Monachino. Vol. 2. Roma 1961, S. 737. 
edorum morem) als ungeeignet für die Liturgie. ${ }^{9}$ Es hieße, dass der dramatische, erregte, exaltierte Vortrag des Textes mit der Frömmigkeit unvereinbar sei. Aber diese Charakteristika sind bereits im 17. Jahrhundert für das Opernrezitativ typisch geworden.

Ein Stück weiter formuliert der Papst seine Einwendungen gegen den weltlichen Vortrag noch klarer. Im Einklang mit dem Jesuiten Jeremias Drexelius (1581-1639) wirft er der gegenwärtigen Musik cantandi genus novum, sed exorbitans, concisum, saltatorium, et parum profecto religiosum, theatro aut choreis convenientius. Mit Jean Harduin (1646-1729), einem anderen Jesuiten, ist sich der Papst einig, dass Kirchenmusik (nach 1700) quid theatrale, aut impudicos amorum, bellorumque classicos modulos nicht enthalten solle, und in Paragraph 9 verurteilt er das Aufführen von Messen und Vespern theatrali more et scaenico strepitu. Bei der Aufzählung der Mängel der Kirchenmusik nennt er mit Giovanni Battista Doni (1595-1647) effeminatum ac levisculum ...canendi modum, affectata prolixaque ac saepe hiulca melismata, molliores, parum decoris cantus und schließlich magnam confusionem vocum ac instrumentorum.

Aus dieser Kritik ist ersichtlich, dass nach der Meinung des Papstes der neue Stil (stile nuovo, seconda prattica) zahlreiche Missstände in die Kirchenmusik eingeführt hätte. Dessen Hauptmängel bestanden vor allem in der exaltierten solistischen Wiedergabe, in dramatischen Exklamationen, Koloraturen und gefälligen, tänzerischen Melodien. Dazu trat noch ein großer, von der Militärmusik übernommene Instrumentalapparat, der die Gesangstimmen übertönte. Das alles aber waren doch typische Züge des hochbarocken Stils. Obwohl die Jesuiten Drexelius und Harduin sowie der gebildete Doni die Missstände der neuen Musik rechtzeitig erkannt hatten, kam die Reaktion des Oberhirtes der katholischen Kirche um hundert Jahre verspätet, als die neue Musik bereits in der Kirchenmusik tief verwurzelt war. Der Papst getraute sich aber nicht, die neue Musik völlig zu verbieten, da es andere geistliche Autoritäten gab, die behaupteten, dass der neue Stil die Frömmigkeit der Gläubigen unterstütze.

In dieser Situation blieb dem Papst nichts anderes übrig, als die Neuigkeiten zu tolerieren und nur ihre Überschreitungen zu mäßigen und abzumildern. Deshalb gab sich Benedikt mit der Forderung zufrieden, dass nur biblische Texte vertont werden sollen und zwar so, dass die Worte gut verständlich seien. Die Vertonung sollte ernst (gravis), eher langsam und von weltlichen und tänzerischen Elementen frei sein. Unter den selben Bedingungen ließ er auch eigenständiges Orgelspiel und instrumentale Musik zu.

Am deutlichsten äußerte sich der Papst zu den für die Verwendung in der Liturgie zugelassenen Musikinstrumenten. Zum Unterschied von der ursprünglichen strikten Anordnung des Mailänder Provinzialkonzils vom 1565 unter der Leitung des heiligen Carlo Borromaeo ${ }^{10}$ nahm Benedikt XIV. am Ende von Paragraph 9 eine versöhnlichere Einstellung zu den Musikinstrumenten ein. Obwohl er ihnen

9 Annus qui, Paragraph 6 und 9.

Sacrorum conciliorum nova amplissima collectio. Ed. Johannes Dominicus Mansi. Firenze. 
relativ wenig Platz einräumte, drückt er sich umso konkreter und strenger über sie aus. Seiner Meinung nach dürften nur jene Musikinstrumente zugelassen werden, die die Gesangstimmen unterstützen oder verstärken. Er zählt sie namentlich auf, wobei er sehr ungewöhnliche, manchmal schwer übersetzbare Benennungen gebrauchte, die wir in Klammern anführen. ${ }^{11}$

Demnach billigte Benedikt XIV.:

barbiton $^{22}$ - Baßlaute

lyras tetracordes-Violinen

fidiculas - Altviolen

tetracordon maius, tetarcordon minus - Violon und Violoncello [?]

monaulon pneumaticum - Fagott

Die genannten Instrumente waren tatsächlich in der Basilica Vaticana seit Ende des 17. Jahrhunderts gebräuchlich. ${ }^{13}$

Dagegen verbot der Papst kategorisch ohne nähere Begründung:

tympana - Pauken

cornua venatoria - Waldhörner

tubas - Trompeten

tibias decumanas - Oboen

fistulas, fistulas parvas - Flöten ${ }^{14}$

psalteria symphoniaca - Harfen

cheles - Lauten ${ }^{15}$

und alle übrigen Instrumente, die die Musik ,theatralisch“ machen würden.

Vielleicht reagierte der Papst auf eigene (schlechte) Erfahrungen aus seiner Wirkungszeit in Bologna. In der Basilica San Petronio leitete damals Giacomo Antonio Perti die Musikkapelle, der an den großen Kirchenfesten bis zu vier Trom-

Tomus 34, p. 57. Das Mailänder Konzil bewilligte für die Kirche nur die der Orgel. Alle übrigen Musikinstrumente hat es verboten.

11 Bei der Übersetzung haben wir uns nach der italienischen Übersetzung der Enzyklika gerichtet.

12 Barbiton, war ein kitharaähnliches altgriechisches Instrument, das in der Neuzeit am ehesten der Theorbe entspräche. Vgl: Sachs, Curt. Real-Lexikon der Musikinstrumente. Berlin 1913, S. 31. Die Baßlaute wurde geläufig als Generalbaßinstrument gebraucht.

13 ROSTIROLLA, Giancarlo, Musici e apparati nella basilica Vaticana per le feste dei santi Pietro e Paolo e della dedicazione dalla fine del XVI al primo quarto del XVII secolo. Analecta musicologica 33, 2004, S. 417-474.

14 Flöten kommen in der katholischen Musik des Barock sehr selten vor. Wir begegnen ihnen erst im letzten Drittel des 18. Jahrhunderts.

15 Das Verbot der Laute ist schwer zu begreifen, da dieses Instrument, insbesondere die Theorbe, seit dem Ende des 16. Jahrhunderts bis zum Anfang des 18. Jahrhunderts in der Generalbassgruppe allgemeine Verwendung fand. 
peten, vier Zincken, vier Oboen und sogar zwei Jagdhörner gebrauchte. ${ }^{16}$ Selbst Pertis Vorgänger, Giovanni Paolo Colonna, engagierte bereits 1687 Trompeter. ${ }^{17}$

Verständlich war das Verbot der Pauken und Trompeten, da diese Instrumente ursprünglich dem Militär und dem Krieg dienten. Auch die Waldhörner kamen aus dem Milieu der blutigen Parforcejagd, die sich beim hohen Adel großer Beliebtheit erfreute, in die Kunstmusik. Bei den Blechblasinstrumenten ließ der Papst merkwürdigerweise die Posaune aus. Vielleicht hielt er dieses seit Jahrhunderten in der Kirchenmusik heimische Instrument für selbstverständlich.

Was dem Papst an den Oboen missfallen hat, ist schwer nachzuvollziehen. Vielleicht erinnerte der Klang dieses Instruments zu sehr an die Schalmei, die gleich wie die Flöten mit dem Tanz verbunden wurde. Während der ersten Hälfte des 18. Jahrhunderts waren jedoch Waldhörner und Oboen zu unentbehrlichen Instrumenten der Kirchenmusik geworden. Der Kapellmeister der Sankt VeitKathedrale in Prag, Kryštof Karel Gayer, bezeichnete 1717 die Oboe als eine besondere Zierde der Musik ${ }^{18}$, und auch ein Bewerber um eine Choralistenstelle an der Olmützer Kathedrale schrieb 1741, dass die Oboe in der modernen Kirchenmusik erforderlich sei. ${ }^{19}$

Die Enzyklika Annus qui ist mit 19. Feber 1749 datiert. Am 1. Mai des gleichen Jahres wurde in Wien jenes Amt errichtet, das die Bewilligung zur Verbreitung von im Ausland herausgegebenen Dokumente, das sogenannte placetum regium, erteilte. Da Annus qui keine politischen Angelegenheiten betraf, gab es keinen Grund, das placetum regium abzulehnen. Trotzdem scheint es, dass diese Enzyklika in der Monarchie lange unbekannt bzw. unbeachtet geblieben ist. Die Prämonstratenser in Hradisko bei Olmütz wussten bereits am 17. Juli 1749, dass diese Enzyklika in Rom publiziert worden war, ${ }^{20}$ doch ist in ihren Diarien darüber kein Wort zu finden, auch über deren Inhalt. Die Annalen der Augustiner Chorherren in Šternberk erwähnen zwar mit 14. März 1750 die Ablässe des Jubiläumsjahres, aber mit keinem Wort die päpstliche Enzyklika. ${ }^{20}$ Daher meinen wir, dass die Enzyklika im Mährischen Bistum nicht publiziert worden ist.

In den Kirchen der ganzen habsburgischen Ländern setzte sich also clangor tympanorum et tubarum bis zum Jahre 1754 ungestört fort. Erst auf eine ausdrückliche Urgenz des Papstes verbot Maria Theresia mit 26. Jänner 1754 die Verwendung der Pauken und Trompeten in den Kirchen und bei Prozessionen in ihrem Herrschaftsgebiet. In Mähren wurde das kaiserliche Reskript am 1. Februar

16 SCHNOEBELEN, Anne. The concerted Mass at San Petronio in Bologna ca 1660-1730. Ph.D. diss. University of Illinois 1966, S. 316.

18 KOSTÍLKOVÁ, Marie. Nástin dějin svatovítského kůru (Abriß der Geschichte des St. Veit Chores). In Štefan, Jiří. Ecclesia Metropolitana Pragensis. Pragae, 1983, S. 26.

19 SEHNAL, Jiří. Hudba volomoucké katedrále v 17. a 18. století. (Geschichte der Musik in der Olmützer Kathedrale im 17. und 18. Jahrhundert). Brno, 1988, S. 94. 
1754 bekannt gemacht. ${ }^{21}$ Das Verbot wurde mit allgemeinem Staunen und Unwillen aufgenommen und es wurde ihm vorgeworfen, dass seine Motive und Hintergründe nicht genannt würden. ${ }^{22}$ Einer ähnlichen Einstellung begegnen wir bei den Augustinern Eremiten in Brünn, die jedoch offen bekannten, dass sie das Verbot an den Festen der ersten Klasse nicht einhalten würden. ${ }^{23}$ Dagegen respektierten die Prämonstratenser in Hradisko bei Olmütz das Reskript Maria Theresias und verzichteten sogar bei der Auferstehungsfeier am 14. April 1754 auf Pauken und Trompeten, quod hoc anno nulla tympana et tubae per prohibitionem Regiam auditae sint. ${ }^{24}$ Der Adel, die Geistlichkeit und insbesondere das Volk in Mähren hielten jedoch auf Trompetenschall und Paukenwirbel so viel, dass eine Kirchenfeier ohne diese Militärinstrumente unvorstellbar gewesen wäre. Die weltlichen Behörden aber überwachten die Einhaltung des kaiserlichen Verbots und ahndeten dessen Überschreitung mit Geldstrafen. ${ }^{25}$

Die päpstliche Enzyklika Annus qui bezog sich in erster Linie auf die liturgische Formen, namentlich auf Messe und Vesper, erst dann in weitesten Sinne auf alle in der Kirche aufgeführte Musik. Die Zeit, in der Annus qui erschienen ist, war zugleich die Blütezeit des Oratoriums, des Sepolcro und der Dramen und Melodramen der Jesuiten wie Piaristen. War Annus qui auch für letztere Formen verbindlich, obwohl sie keine gottesdienstlich Funktion hatten? Wenn wir die Kirchenmusik des 18. Jahrhunderts als Ganzes betrachten, scheint es dass diese päpstliche Enzyklika die Musik in der Liturgie nicht wesentlich beeinflusst hatte. Sicherlich haben die Komponisten der Klassik den gebührenden Respekt zu dem liturgischen Text genau eingehalten, aber in der musikalischen Rede sich frei verhalten. Deshalb wird wohl Annus qui die Musik der Oratorien und Sepolcri weniger beeinflusst haben, da diese Formen keine Gattungen der liturgischen Musik darstellten, sondern eher ein Konzert in der Kirche waren. Vielleicht werden wir in unserer Konferenz über diese Frage mehr erfahren.

Jiří Sehnal (jiri.sehnal@email.cz), Katedra muzikologie, Univerzita Palackého v Olomouci.

21 Vgl. SEHNAL, Jiř́. Trubači a hra na přirozenou trompetu na Moravě v 17. a 18. století. (Trompeter und Spiel auf der Naturtrompete in Mähren im 17. und 18. Jahrhundert). 1. Teil. Acta Musei Moraviae - scientiae sociales 73, 1988, S. 196-198.

28. 2. 1754. ... Regina nostra inhibeat tympana in Ecclesiis et processionibus producenda, quo motivo et ratione nihil additum, Annales Canoniae Sternbergensis. 5. 3. 1754... Missa prima vice sine tympanis et tubis. MZA, E 5, kart. 22, kn. 18.

SEHNAL, Jiří. Hudba u brněnských augustiniánů v 18. století (Musik bei den Augustinern in Brünn im 18. Jahrhundert). Hudebni věda 20, 1983, S. 238.

24 Liber octavus Annalium Praemonstratensium, MZA, E 54, kart. 22, kn. 17. Ähnlich bei den Augustiner Chorherren in Šternberg wie in Anm. 20.

25 PEŘINKA, František Václav. Kotly a trubky v kostele (Pauken und Trompeten in der Kirche). In Od Horácka k Podyjí 1934, S. 71-73. 


\section{ABSTRACT \\ PIETY AND MUSIC}

This paper is dedicated to explaining how the term Piety was used with respect to Music. The most important document concerning liturgical music within the Roman Catholic Church in the 18th century was the Encyclica Annus qui, published by Pope Benedikt XIV in 1749 for the jubilee year of 1750. This document represents the somewhat delayed official reaction of the church to the so-called "stile nuovo" and opera. Its aim was to weaken the impact of musical innovations developed within secular music on liturgical music. This document was apparently not published in the Habsburg Empire in 1750, because the prohibition of trumpets and kettle drums in church music was not declared by the empress Maria Theresia until 1754.

\section{Key words}

Sacred music, Roman Catholic Church, Encyclica Annus qui

\section{Bibliography}

FELLERER, Karl Gustav. Die Enzyklika Annus qui des Papstes Benedikt XIV. In Geschichte der katholischen Kirchenmusik. Band 2. Kassel 1976.

Katechismus katolické cirkve, Praha, 1995.

KOSTÍLKOVÁ, Marie. Nástin dějin svatovítského kůru (Abriß der Geschichte des St. Veit Chores). In Štefan, Jiří. Ecclesia Metropolitana Pragensis. Pragae, 1983.

MATTEUCCI, Benedetto. Il papato di fronte dell' assolutismo e al giurisdizionalismo (1605-1774). In I papi nella storia a cura di Pio Paschini-Vincenzo Monachino. Vol. 2. Roma 1961.

PEŘINKA, František Václav. Kotly a trubky v kostele (Pauken und Trompeten in der Kirche). In Od Horácka k Podyji 1934.

ROSTIROLLA, Giancarlo, Musici e apparati nella basilica Vaticana per le feste dei santi Pietro e Paolo e della dedicazione dalla fine del XVI al primo quarto del XVII secolo. Analecta musicologica 33, 2004.

Sacrorum conciliorum nova amplissima collectio. Ed. Johannes Dominicus Mansi. Firenze. Tomus 34.

SACHS, Curt. Real-Lexikon der Musikinstrumente. Berlin 1913.

SEHNAL, Jiř́i. Hudba u brněnských augustiniánů v 18. století (Musik bei den Augustinern in Brünn im 18. Jahrhundert). Hudebni véda 20, 1983.

SEHNAL, Jiří. Hudba v olomoucké katedrále v 17. a 18. století. (Geschichte der Musik in der Olmützer Kathedrale im 17. und 18. Jahrhundert). Brno, 1988.

SEHNAL, Jiří. Trubači a hra na přirozenou trompetu na Moravě v 17. a 18. století. (Trompeter und Spiel auf der Naturtrompete in Mähren im 17. und 18. Jahrhundert). 1. Teil. Acta Musei Moraviae - scientiae sociales 73, 1988.

SCHNOEBELEN, Anne. The concerted Mass at San Petronio in Bologna ca 1660-1730. Ph.D. diss. University of Illinois 1966. 
